\title{
Toward Ubiquitous Communication Platform for Emergency Medical Care
}

\author{
Kenichi ISHIBASHI $^{\dagger a)}$, Naoto MORISHIMA ${ }^{\dagger}$, Nonmembers, Masayuki KANBARA $^{\dagger}$, \\ Hideki SUNAHARA ${ }^{\dagger, \dagger}$, Members, and Masami IMANISHI ${ }^{\dagger \dagger}$, Nonmember $^{\dagger}$
}

\begin{abstract}
SUMMARY Interaction between emergency medical technicians (EMTs) and doctors is essential in emergency medical care. Doctors require diverse information related to a patient to provide efficient aid. In 2005 , we started the Ikomal19 project and have developed a ubiquitous communication platform for emergency medical care called Mobile ER. Our platform, which is based on wireless internet technology, has such desirable properties as low-cost, location-independent service, and ease of service introduction. We provide an overview of our platform and describe the services that we have developed. We also discuss the remaining issues to realize our platform's actual operation.

key words: emergency medical care, mobile network, Mobile IPv6, network mobility
\end{abstract}

\section{Introduction}

In emergency medical care, information about patients and their conditions greatly affects the quality of medical treatment. Immediacy, amount, and accuracy of medical information are essential for improving the quality of medical treatment. In current information transmission systems, an exclusive line is commonly used to transmit medical information to doctors from ambulances. For example, information acquired from medical equipment is transmitted using cellular phones. However, information transmission systems currently used in emergency medical treatment have some drawbacks. First, supporting diverse information is difficult. Appropriate medical treatment requires exchanging such diverse information as a patient's clinical history, vital signs, and images of the affected areas. However, most services currently used to transmit medical information require separate exclusive lines for each bit of information. To support new medical information, we need more exclusive lines, but this increases the system cost. Second, information and its transmission lines are strongly bound. Since most current medical information transmitting services do not provide a function for switching transmission lines, a service will not be available when the corresponding transmission line is out of service. Third, existing information

Manuscript received September 30, 2008

Manuscript revised December 16, 2008.

${ }^{\dagger}$ The authors are with the Graduate School of Information Science, Nara Institute of Science and Technology, Ikoma-shi, 6300192 Japan.

${ }^{\dagger}$ The author is with the Graduate School of Media Design, Keio University, Yokohama-shi, 223-8521 Japan.

${ }^{\dagger \dagger}$ The author is with Nara Prefectural Gojo Hospital, Gojo-shi, 637-8511 Japan.

a)E-mail: kenich-i@is.naist.jp

DOI: 10.1587/transcom.E92.B.1077 services assume fixed point-to-point communication. For example, if a service uses digital cellular access as the transmission line, transmitting information to multiple destinations is difficult. Let us consider the situation in determining the most suitable hospital for a patient. In this situation, it is desirable that the information on the patient's condition can be transmitted to all of the candidate hospitals, but it is difficult when the service uses a fixed point-to-point communication line. Fixed point-to-point communication is insufficient for emergency medical care and the service should provide data transmission to multiple destinations.

The essential problem, including the above drawbacks, is the lack of integration of both information and communication lines. A communication platform is needed for emergency medical care that solves the above problems. In 2005, we started a joint project to develop an efficient communication platform for emergency medical care. This project is called the Ikomal19 project [1], which is affiliated with the Nara Institute of Science and Technology and the Nara Prefectural Nara Hospital. The Ikoma1 19 project has developed a communication platform between doctors and EMTs for emergency medical care called Mobile ER, which is based on wireless internet technology. Our platform is approaching practical use a total of three demonstration experiments have already been conducted. We show the design and the implementation of our platform, and also describe some developed services running on it.

\section{Background and Technology}

Since most emergency medical care activity happens far from hospitals, a communication method between hospitals and ambulances is needed. As we will describe in Sect. 3 in detail, our Mobile ER communication platform is based on wireless internet technology. In our platform, an ambulance is connected to the Internet by a wireless data link, and then an emergency medical technician (EMT) in the ambulance and a doctor in a hospital communicate via the Internet.

Outstanding technological advances in mobile computing and internet technology are motivations for developing our Mobile ER platform. Wireless data link technology, mobility support for Internet Protocols (IPs), and wearable computing are key technologies for Mobile ER platforms. 
Table 1 Comparison characteristics of WLAN and PHS

\begin{tabular}{c|cc}
\hline & WLAN & PHS \\
\hline \hline bandwidth & $\begin{array}{c}\text { broadband } \\
\text { limited }\end{array}$ & $\begin{array}{c}\text { narrowband } \\
\text { nationwide }\end{array}$ \\
\hline
\end{tabular}

\subsection{Wireless Internet Technology}

In Japan's current mobile environment, many different kinds of wireless data links can be used to connect to the Internet, including wireless LAN (WLAN), 3G cellular phones, Personal Handy-phone Systems (PHSs), and High-Speed Downlink Packet Access (HSDPA). These data links have different characteristics; a mobile node detects the appropriate data link depending on the situation. Consider a situation where a mobile node has both WLAN and PHS interfaces. As shown in Table 1, PHS can be used in a wide area but with a lower bandwidth and a large delay, while WLAN provide high bandwidth with limited coverage area. In this situation, the mobile node adapts these data links to acquire efficiency and availability. If a mobile node is in the WLAN's range, it will use the WLAN as a data link to receive large bandwidth. When a mobile node breaks away from the WLAN's range, it will check for connectivity to a PHS and try to switch its data link from WLAN to PHS since PHS still seems to be available.

\subsubsection{Mobility Support for IP}

In our platform, an ambulance is connected to the Internet by a wireless data link and communicates with a correspondent node (CN) (e.g., a doctor's PC) through the Internet. Since an ambulance travels anywhere, it must deal with inter-domain handover, which can change the IP address of the ambulance at any point in time. Two problems typically surface when the ambulance changes its IP address. First, transport layer or higher layer connections are disconnected. If the ambulance changes its IP address while transmitting information, this information will be lost. Second, the CN will not be able to distinguish the ambulance because the $\mathrm{CN}$ can only distinguish the ambulance by its IP address. The CN cannot connect to the ambulance while the ambulance's mobile node (MN) is being notified of its new IP address.

The Internet Engineering Task Force (IETF) developed and standardized technology to prevent the above problems: Mobile IPv6 [2] for a single MN and Network Mobility (NEMO) Basic Support [3] for a mobile network.

(1) Mobile IPv6

Mobile IPv6 (MIPv6) provides mobility transparency for a single MN. Mobility transparency is the property that guarantees reachability and connectivity for an MN. The basic concept of MIPv6 is using a permanent IP address, which is called a Home Address (HoA) provided by a Home Agent (HA). An HA is placed in the home network of the MN and acts as an intermediate node between the $\mathrm{MN}$ and the $\mathrm{CN}$.

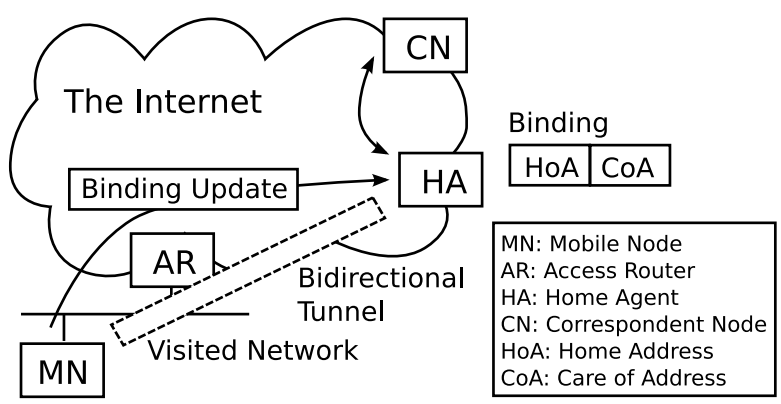

Fig. 1 Conceptual architecture of Mobile IPv6.

Figure 1 shows an overview of how MIPv6 works. The $\mathrm{MN}$ sends a binding update (BU) message to the HA to notify the whereabouts of the MN. The BU message includes a Care of Address (CoA), which is provided by the access router (AR) on the visited network, and a HoA of the MN. When the HA receives the BU message, the HA creates or updates a binding cache entry that associates the HoA with the CoA and then creates a bidirectional tunnel between the $\mathrm{MN}$ and the HA. All packets are sent from the MN to the $\mathrm{CN}$ through this tunnel. Because the HoA is used for the source addresses of these packets, the $\mathrm{MN}$ and $\mathrm{CN}$ can communicate with each other even though the CoA has changed.

MIPv6 supports route optimization in which the MN communicates with the $\mathrm{CN}$ directory; however, we do not describe the route optimization mechanism because route optimization does not support network mobility basic support, which is the key technology of our communication platform, which we will describe later.

\section{(2) NEMO Basic Support}

An $\mathrm{MN}$ is likely to form a network under itself called a mobile network when it is a vehicle [4]. Network Mobility (NEMO) Basic Supports are designed as extensions to MIPv6 to provide mobility transparency for mobile networks. With NEMO, an MN is called a mobile router (MR) since the MN acts as the gateway of the mobile network to provide connectivity to the Internet with mobile network nodes (MNNs). The BU message in NEMO provides additional information, including a mobile network prefix (MNP), which is a network prefix of mobile networks advertised from the MR to the MNNs. The HA maintains a relationship between the MR's HoA and the MNP, so each packet sent from the $\mathrm{CN}$ to an $\mathrm{MNN}$ is appropriately forwarded by the HA and arrives at the MNN through the bidirectional tunnel.

\subsection{Wearable Computing}

Advances in wearable computing technology are another motivation behind the development of our Mobile ER platform. Through an EMT who is working inside or outside of an ambulance and is wearing a wearable computer, various kinds of information can be processed and the EMT can cooperate with a doctor at any time. 
In recent years, many sophisticated portable computers have been developed, such as Laptop PCs, personal digital assistants (PDAs), and smartphones. They can be used as wearable computers. Even though these portable computers are small, lightweight, and are power-saving devices, they have high processing capability. This enables them to handle heavily loaded processes and provide various services to anyone who is equipped with a wearable computer. For example, live encoding and streaming with H.264 on laptop PCs will be possible.

The mobility of wearable computers is obviously important for emergency medical care. As typified by Bluetooth and ZigBee, personal area network (PAN) protocols have improved wearable computing technology. PAN enhances the mobility of wearable computing because no cables are needed to connect the input/output devices. With PAN, a head mounted display (HMD), which is a display device designed to be mounted on the head, a headset and a webcam can be connected to the wearable computer without sacrificing mobility.

\section{Mobile ER Platform}

Our ultimate goal is to form a ubiquitous communication platform for emergency medical care to enhance the quality of life-saving activities. To form a feasible communication platform, we must investigate the requirements for such technology. Once the requirements are determined, we can concentrate on designing and implementing the platform to satisfy them.

\subsection{Requirements}

In emergency medical care, the main source of information is the neighborhood of patients. The information derived from the neighborhood of patients should be shared by persons involved, such as doctors, EMTs, and an administrator of an ambulance, for appropriate administration of emergency medical care. Patients can be anywhere, and ambulances and EMTs can go almost anywhere. Moreover, locations of doctors who give EMTs instructions could change depending on the situation; a doctor who gives instructions will be at the hospital assigned to the patient. However, such a hospital may change according to the situation. Since there are various possible combinations of sources and destinations of the information, the platform should provide not only integration of the communication lines but also a location-independent service in a cost effective way.

There are many other important aspects for an appropriate communication platform such as extensibility, efficiency, availability, and robustness. The following states the requirements to be fulfilled with the platform.

- Flexibility to support diverse information: The information exchanged between a doctor and an EMT is often diverse: images, audio, video streams, text data, electrocardiogram (ECG) data, and a patient's clinical history. Since this information has different characteristics, appropriate transmission methods depend on the information characteristics. Some information (e.g., text data) must be accurately transmitted, while other information (e.g., video streams) should be transmitted quickly at the cost of accuracy. For example, text data or a document file could have a certain file format and it could be corrupted if the data does not accuracy transmit. While video streams are typically tolerant of some data loss but they should be transmitted in real time. The communication platform must have flexible transmission methods to support appropriate data transmission.

- Universal connectivity: Since ambulances travel almost anywhere, the platform should provide connectivity at any place at any time. This is especially true for important information such as ECG data might be useless when the platform lacks connectivity because these types of data represent patient's rel-time conditions. Doctors cannot give appropriate medical directions when this data is delayed. Other information, which is not always time sensitive, still should be transmitted as soon as possible to inform doctors of the latest information.

- Location independent service: In emergency medical care, a doctor is typically in a hospital and an EMT is typically in an ambulance, although both might be in different places. A doctor may be away from a hospital when an emergency call is made. A doctor in a hospital may be on his or her rounds and may not go to the operating room immediately. An EMT might be in the neighborhood of an ambulance at an earlier stage of the emergency medical care. Therefore, the platform must have the capability to transmit information anywhere to enhance the quality of emergency medical care.

- Device and data link independent services: At present, many emergency medical information systems depend on specific devices and data links. In such information systems, devices and data links are inseparable from the information transmission service. This might be a potential drawback. When an ambulance leaves the service area of the data link, the service using that data link will no be longer available. In addition, since wireless communication technology is constantly improving, the data link currently being used may soon become obsolete. If the service and the data link are inseparable, it will increase the cost of introducing a new medical service, which will require a vast amount of bandwidth. By avoiding dependence between service and data links, the platform can resolve these drawbacks.

- Easy installation of new services: In an ambulance, information is generated by many devices such as medical equipment and global positioning systems (GPSs). The platform should provide a common and easy way to connect these devices.

- Dependability: Dependability is crucial for the plat- 


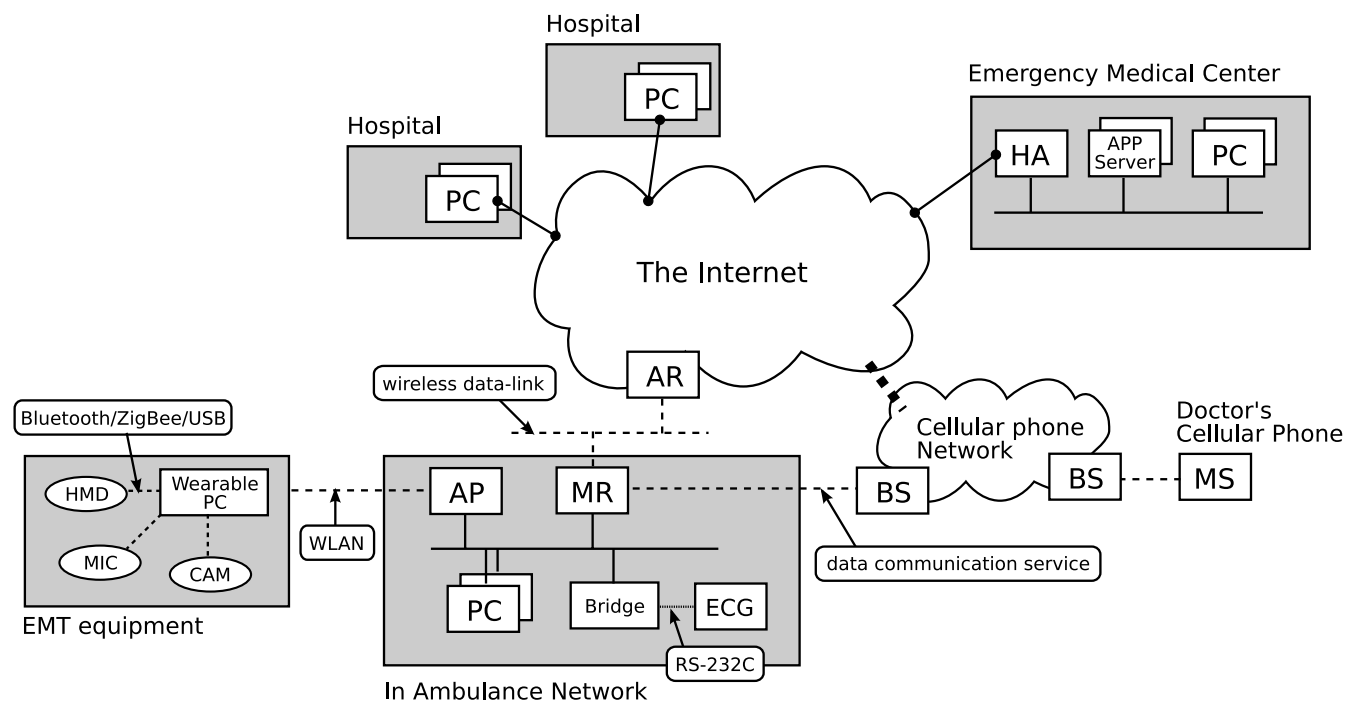

Fig. 2 Conceptual architecture of Mobile ER platform.

form. Wireless data links often lack connectivity and stability; however, the platform should provide appropriate availability of services whenever possible. It must minimize the impact of the lack of connectivity and the stability of the data link.

- Security: In greater or lesser degrees, any information treated in emergency medical care is related to a patient. Such information is considered private information. Therefore, the platform must protect this private information, especially when the platform handles or transmits it.

\subsection{Design}

Based on the above requirements, we designed a communication platform for emergency medical care called the Mobile ER that uses wireless internet technology for the communication scheme and wearable computing technology for the EMT equipment. In this section, we outline the design overview and then describe the details of the platform design.

\subsubsection{Design Overview}

Figure 2 illustrates the conceptual architecture of the Mobile ER platform. We adopt internet technology as the communication infrastructure because this technology has appropriate properties for satisfying most of the requirements mentioned above. In 3.2.2, we will discuss why we adopted internet technology as the communication infrastructure.

The platform is divided into four main components: an emergency medical center network, in-ambulance network, EMT equipment, and service clients. An emergency medical center is placed by the organization, which is responsible for controlling ambulances. In Japan, the fire defense divisions of local governments are the organizations that operate ambulances. In the platform, the organization must place a network; the network contains an HA to provide mobility transparency for an in-ambulance network, and also contains application servers to enhance the quality of services provided by the platform. An in-ambulance network is a network, which is located in an ambulance and connects several devices and PCs to each other in the ambulance. These devices and PCs could be a component of certain services provided by the platform. To provide connectivity to the Internet, an MR is also connected to the in-ambulance network. EMT equipment consisting of several wearable computing devices such as a wearable PC, an HMD, a microphone, and a webcam. An EMT puts on the EMT equipment to communicate with doctors. Service clients are the clients of services provided by the platform. Since the platform based on internet technology, various kinds of devices which connect to the Internet could be a service client. An doctor's PC in a hospital typically could be a service client but not limited to that. Sometime the cellular phone of a doctor could be a service client when the doctor is away from the hospital.

Each component of the platform is connected with the others by the Internet except for the EMT equipment. The EMT equipment connects to an in-ambulance network and is connected to others through an in-ambulance network. Since an in-ambulance network's mobility transparency is provided using NEMO, all components can constantly communicate with each other regardless of the ambulance's location. This means that the platform can provide locationindependent service as long as the ambulance remains connected to the Internet.

In the platform, services are used as internet applications. The platform has the flexibility of developing services. These services can be constructed in various architectures because each component of the platform can communicate with each other. 


\subsubsection{Communication Technology and Services}

As mentioned above, our platform uses the Internet, which is based on TCP/IP protocol suites, as the communication infrastructure, and services on the platform are used as TCP/IP applications. Internet technology has appropriate properties to satisfy most of the requirements described in 3.1, with some few exceptions. In this section, we will describe the appropriate properties first, and then describe properties of internet technology that not appropriate for the requirements mentioned the previous section.

The first advantage of internet technology is that it is open to the public and is widely used. This is a cost advantage. Since many applications and libraries have been developed that use the Internet, we can exploit them to develop a new service on our platform. In addition, service clients do not need to prepare extra equipment. Most TCP/IP applications, especially web-based applications, will work in almost all communication nodes. For example, people can browse web pages using their cell phones. This advantage makes it possible to develop device independent services. Furthermore, commonly used methods of developing $\mathrm{TCP} / \mathrm{IP}$ applications can be applied to emerging services. When introducing a new service, we simply develop its specific components as a standard TCP/IP application.

The second advantage is that the TCP/IP protocol suite can be replaced. The TCP/IP protocol suite is divided into four layers in a hierarchical structure. Since a minimal relationship exists between layers, a protocol or technology that composes a layer can be easily switched. For example, consider the physical layer. The data link currently used to connect to the Internet can switch to a new data link without affecting the other layers. Since the change in the data link does not affect services running on the platform, the platform can provide a data link-independent service. Next, consider the transport layer. The TCP/IP protocol suite has different types of transport protocols such as TCP and UDP. A service selects an appropriate transport protocol depending on the characteristics of the information sent by the service. For example, a video streaming service will use Realtime Transport Protocol (RTP) [5] with UDP, while a textbased service will use TCP.

In contrast with the above advantages, the internet technology is currently not all good for a few requirements. In particular, dependability is not entirely guaranteed on the Internet. However, the current Internet has higher reliability in general. It is not likely that the Internet loses its functionality easily. It must also take care of security. Since our platform uses the Internet as communication infrastructure, it must protect privacy. An MR works as required by NEMO specifications; all traffic sent through the bidirectional tunnel between the MR and the HA is encrypted by IPSec [6]. However, a communication between an $\mathrm{HA}$ and a $\mathrm{CN}$ is not encrypted by default. Therefore, the service must use security technology such as TLS [7].

\subsubsection{Emergency Medical Center Network}

An emergency medical center network has two roles, a home network of the MR in the ambulance and an intermediate service network. The network must have an HA and may have several application servers and PCs.

The HA, which is the home agent of the ambulance, collaborates with the MR in the ambulance to provide mobility transparency to both the MR and the MNNs. The HA maintains the ambulance's current location. When the HA receives a packet intended for the MR or the MNNs in the ambulance, the HA forwards it to the MR.

Application servers could be located in the network depending on the demands of the service provided by the platform. Their roles reflect what service is needed. Typically, application servers act as proxy nodes. A proxy server improves the service availability and dependability. The connectivity and stability of the ambulance may deteriorate due to its movement. The service may not be available when the ambulance lacks connectivity in the absence of a proxy server. In contrast, when a proxy server is available and retrieves the information from the ambulance, the application server provides the information to clients instead of the source device of the information. There are many other applications for the application server: cache, data conversion, relay, and so on.

\subsubsection{In-Ambulance Network}

Ambulances contains several different kinds of devices, sensors, and computers that should be connected to the Internet to provide diverse information to clients as services. However, these devices may not have enough processing capacity to individually support mobility transparency. We therefore introduced an MR to the ambulance. The MR forms a network and provides mobility transparency to the network with NEMO. The MR, which has multiple wireless interfaces, selects these interfaces to connect to the Internet depending on the situation because it has mobility transparency provided by NEMO. Note that the wireless interfaces can be replaced. The platform easily switches its data link to a new one without any impact on the other components, when high-performance wireless data link technology is available.

There is the flexibility in configuring an in-ambulance network because the network is the same as a general LAN. We can choose the appropriate design of the network according to the requirements of services to be provided. For example, if an ambulance has few devices, we will put only one PC into the network to correct and aggregate the information from these devices. If we try to provide a heavily loaded service, such as streaming video, we need to put a dedicated server into the network for that service.

Since some medical equipment do not have a network interface, the equipment cannot be directly connected to the network. However, such equipment may have other inter- 
faces, such as an RS-232C and a USB, which can be used to construct bridge devices to connect the equipment to the network. A bridge device has both network and other input/output interfaces and can work as a bridge between these interfaces.

An EMT often works outside the ambulance; typically, he or she will be in the neighborhood of an accident scene helping the injured. To provide connectivity for an EMT who works outside of the ambulance, we install an access point (AP) in the ambulance network. The AP acts as a bridge and provides wireless connectivity from the inambulance network to EMTs.

\subsubsection{EMT Equipment}

The main component of the EMT equipment is a wearable computer that provides several functions for an EMT. To provide connectivity to the Internet, the wearable computer has a WLAN interface and connects to the AP in the inambulance network. The wearable computer also has interfaces such as Bluetooth, ZigBee, and USB, that are used to connect input/output devices. An HMD, a microphone (MIC), and camera (CAM) devices are connected by these interfaces for interaction with doctors.

\subsubsection{Service Clients}

The role of service clients depends on the services used by doctors or other medical staff, and they should communicate with any components of the platform. For example, an ECG information transmission service client receives ECG information from ECG equipment in an ambulance and graphically display it. We can also develop a group chatting service, in which service clients communicate with each other, to provide a mechanism for remote consultation.

Since services on our platform are provided through the Internet, service clients can be anywhere as long as they have connectivity to the Internet, if a client has proper authority to use the service. In addition, no specific equipment is needed to use the services. This means that our platform provides a location/device-independent service. A service client could be a commonly used PC in a hospital, a doctor's cellular phone, or a communication terminal in an emergency medical center.

\subsection{Current Implementation}

Many components of our platform can be replaced because we designed the platform as a device/data link-independent platform. The main specifications of the current implementation are listed in Table 2. MIPv6 and NEMO are supported by SHISA [8], which is an MIPv6/NEMO implementation running on several BSD-based OSs developed by the Widely Integrated Distributed Environment (WIDE) Project [9]. The MR uses the following wireless data links: IEEE802.11b/g, b-mobile [10], and emobile [11]. When the MR is in a WLAN coverage area, it uses a WLAN as the
Table 2 Current implementation specifications.

\begin{tabular}{c|l}
\hline \multicolumn{1}{c}{ Mobile Router (MR) } \\
\hline \hline OS & NetBSD2.0 + SHISA \\
custom made \\
Network IF & IEEE802.11b/g, b-mobile, e-mobile \\
\hline \multicolumn{2}{c}{ Home Agent (HA) } \\
\hline \hline OS & FreeBSD 5.4-RELEASE + SHISA \\
HW & Dell PowerEdge \\
\hline \multicolumn{2}{c}{ Wearable Computer } \\
OS & Windows XP SP2 \\
Network IF & Sony Vaio type U \\
I/O IF & USBE802.11b/g Bluetooth \\
HMD & Micro Optical SV-6 \\
Camera & custom made \\
Microphone & Bluetooth MIC \\
\hline
\end{tabular}

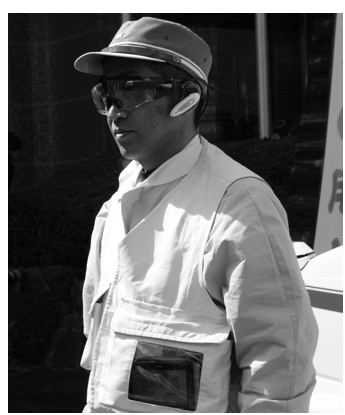

Fig. 3 EMT equipment.

data link. Once the MR moves outside a WLAN coverage area, the MR switches its data link to b-mobile or emobile.

Figure 3 shows the EMT equipment. The EMT wears goggles with a built-in camera that always follows the direction of the EMT's eyes. The HMD, which is mounted on the goggles, displays diverse information to the EMT.

\section{Mobile ER Services}

We have developed the following three services on our platform: still image transmission, ECG information transmission, and video interaction. In this section, we describe their design and implementation. These services can be easily built into each other because they share common communication platforms and are based on extensively used internet technology. In fact, the video interaction service uses the ECG information transmission service as a component.

\subsection{Still Image Transmission Service}

At an early stage in our project, we developed a still image transmission service that periodically transmits still images of the EMT's viewpoint to medical institutions. This service works as follows:

- The wearable computer takes images with the camera embedded in the goggles at regular (15 seconds) intervals and sends them to the image processing PC in the 


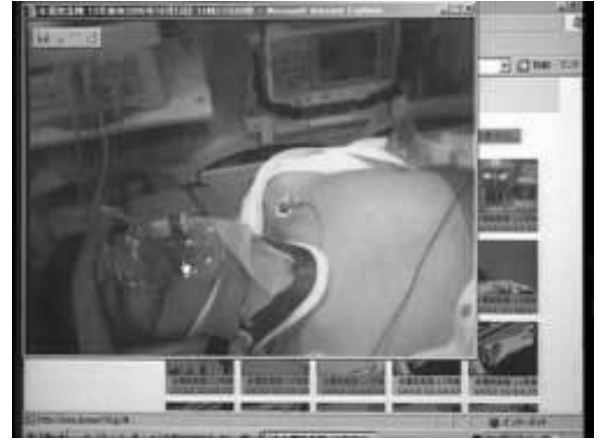

Fig. 4 Screenshot of still image transmission service.

ambulance via the WLAN.

- After receiving an image, the PC generates and stores a thumbnail image as well as the original image. The $\mathrm{PC}$ also notifies the application server in the emergency medical center network that a new image has arrived.

- The application server does not immediately acquire images from the PC after being notified; instead, the server creates a request message which is a message that indicates the server should acquire the thumbnail image from the PC later. Then, the server appends the request message to the request queue, which holds previous request messages, to acquire the thumbnail image triggered by the notification. This behavior is designed with the bandwidth limitation of the ambulance's data link in mind.

- If a request message is found in the request queue, the application server opens it and acquires the image based on the message.

- A doctor can view these images on her PC using a web browser from the application server.

Figure 4 shows a screenshot of the web interface of this service. The web interface lists the previously taken thumbnail images. If the doctor would like to study the image more closely, she can request the server to acquire the original. When the server receives the request, it immediately sends the original image if the server has already acquired it; otherwise, the server acquires the original image from the image processing $\mathrm{PC}$ in the ambulance and sends it to the doctor's PC.

\subsection{ECG Information Transmission Service}

One of the most helpful pieces of information in emergency medical care is an ECG. This service distributes ECG information to a doctor. There are three desired properties for distributing ECG information: immediacy, security, and availability.

An ECG describes a patient's momentary condition. If the service takes too much time to transmit the information, this information may become meaningless. Therefore, the doctor should receive the information as soon as it is gathered. In addition, the ECG information must be encrypted because it is considered personal. To satisfy immediate and

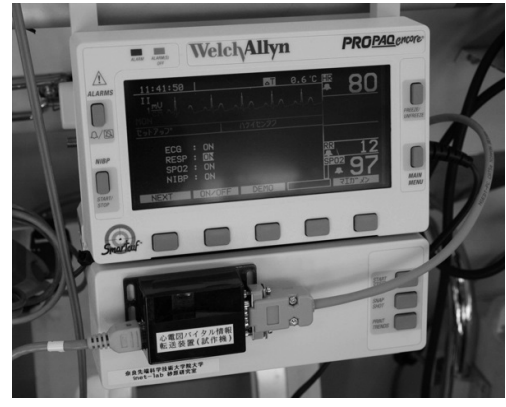

Fig. 5 ECG equipment and bridge device.

secure information transmission, the service uses the Secure Real-time Transport Protocol (SRTP) [12].

To enhance availability, the service locates the proxy server in the emergency medical center network. The proxy server is the sole client that receives the ECG information from the ambulance and is a proxy for other clients. Other clients (e.g., a doctor's PC) are connected to the proxy server to receive the ECG information.

The proxy server provides many ways to transmit the ECG information. Since clients can connect by SRTP, TCP, or HTTP, we developed different kinds of clients. In fact, we developed several clients: a standalone application using SRTP, a Java applet using TCP and a DoJa [13] application, which is used on NTT DoCoMo cellular phones, using HTTP. This feature also enhances the service's availability. Even if a doctor is away from the hospital, he can view the ECG information using the DoJa application with a cellular phone.

Because current ECG equipment does not have a network interface, we prepared a bridge device to connect it to the in-ambulance network. Figure 5 shows the ECG equipment and our current bridge device, which also acts as a SRTP server to transmit ECG information to the proxy server.

\subsection{Video Interaction Service}

We also developed a video interaction service that supports the cooperated emergency medical activity. This service was developed with Adobe's Flash Video (FLV) technology for easy installation. Most modern web browsers support Adobe's Flash and FLV by a plug-in component, so we only have to prepare a browser and a plug-in for a PC.

The following is a typical processing flow of this service. The streaming server is in the emergency medical center network. Both a doctor and an EMT publish the live streaming video with a camera and headset to the streaming server. They subsequently connect to the streaming server as subscribers to receive other live streams. Accordingly, the doctor and the EMT can communicate by video and voice. These live streams have some a few, but non-negligible, delays due to encoding and decoding of the videos. At the present time the delays are approximately less than $1 \mathrm{sec}-$ ond. These delays somewhat complicate the communica- 


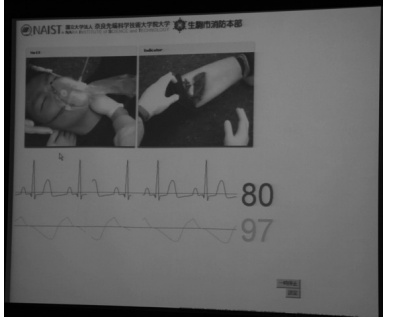

(a) Screenshot of doctor-side interface

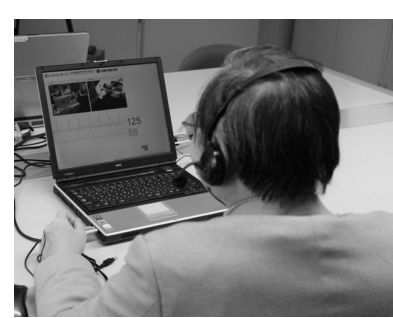

(b) Doctor using service
Fig. 6 Video interaction service.

tion between the doctor and the EMT, but this service is still useful. For example, the doctor can observe a patient's condition.

Figure 6 shows an example of a doctor using the video interaction service. The left image shows the doctor-side web-based interface. There are video streams published by an EMT and ECG information. A doctor can precisely indicate to the EMT what she would like to check. The still image, located in the right of the video stream, shows what the doctor is analyzing. The still image also displays the HMD of the EMT. The ECG information is provided by the ECG information transmission service described above.

\section{Issues}

We introduced the Mobile ER platform to the Ikoma City Fire Department on a trial basis. We also conducted some public demonstration experiments. The Mobile ER platform steadily performed close to practical use. However, several issues remain to be solved. In this section, we discuss how they relate to the practical use of the Mobile ER platform.

\subsection{Complexity of Operation}

The maturity of the platform's current implementation is insufficient for practical use. The platform, which currently requires an operator with expert knowledge of internet technology, must be easy to operate in the future without such an expert operator. The EMT equipment also needs improvements in comfort and start-up time. In emergency medical care, an EMT must act rapidly. The current EMT equipment takes too much time at start-up. This delay is not acceptable for emergency medical care.

\subsection{Performance}

One major platform issue is the performance of the wireless data links. We developed several services, as described in Sect. 4. However, no data link provides both enough bandwidth and broad coverage area to fulfill the services required. Although WLAN has large bandwidth, it is not likely to be available every where. Consequently ambulances would usually use b-mobile or e-mobile, but these data links are inadequate for such services as video interaction. Next generation wireless technology, such as Mobile
WiMAX and Fourth-Generation wireless communication, is a prospective candidate for data links.

\subsection{Reliability}

It is difficult to say whether the current implementation of the platform is reliable enough. The platform remains in a supporting and enhancing system for emergency medical care.

\subsection{Security}

Most information through a platform's personal information. In our platform, information through the MR and the HA will be protected by IPSec [6]. However, the communication channel between the HA and service clients is not always protected. Therefore, each service must protect its information separately. In addition, this information must be protected not only by the communication platform but also by the devices that act as the service clients. In other words, information stored or used in service clients should be protected and encrypted. For the purpose of information protection through the communication channel, a service should be able to adopt IPSec or TLS [7], and for the purpose of encryption of information, a service will be able to use various encryption algorithms.

\section{Conclusion}

We described our Mobile ER platform, which was designed and developed for ubiquitous communication platforms for emergency medical care. The platform is based on wireless internet and wearable computing technologies. Appropriate properties derived from these technologies include flexibility, availability, and low-cost. We developed several services on the platform and introduced it into an actual environment for a trial. Currently, our platform only provides secondary services for emergency medical care; however, it will become a comprehensive communication platform for emergency medical care with advances in wireless and internet technology in the future.

\section{Acknowledgements}

We would like to thank Masami Yamada and Shigeki Kagebayashi of the Ikoma City Fire Department and Ikoma councilman Kazuhisa Shiramoto. We also would like to thank the Nara Prefectural Nara Hospital, Welch Allyn Japan, EZIX Inc., the Internet Automobility Laboratory, Inc., WIDE Project, and the InternetITS Consortium.

\section{References}

[1] “IKOMA119 Project," http://www.ikoma119.jp/

[2] D. Johnson, C. Perkins, and J. Arkko, "Mobility support in IPv6," RFC 3775 (Proposed Standard), June 2004.

[3] V. Devarapalli, R. Wakikawa, A. Petrescu, and P. Thubert, "Network mobility (NEMO) basic support protocol," RFC 3963 (Proposed Standard), Jan. 2005. 
[4] T. Ernst, K. Uehara, and K. Mitsuya, "Network mobility from the InternetCAR perspective," Advanced Information Networking and Applications, 2003. AINA 2003. 17th International Conference on, pp.19-25, 2003.

[5] H. Schulzrinne, S. Casner, R. Frederick, and V. Jacobson, "RTP: A transport protocol for real-time applications," RFC 3550 (Standard), July 2003.

[6] S. Kent and K. Seo, "Security architecture for the Internet protocol," RFC 4301 (Proposed Standard), Dec. 2005.

[7] T. Dierks and E. Rescorla, "The transport layer security (TLS) protocol version 1.2," RFC 5246 (Proposed Standard), Aug. 2008.

[8] "SHISA." http://www.mobileip.jp/

[9] “WIDE Project." http://www.wide.ad.jp/

[10] “b-mobile." http://www.bmobile.ne.jp/

[11] “EMOBILE.” http://emobile.jp/

[12] M. Baugher, D. McGrew, M. Naslund, E. Carrara, and K. Norrman, "The secure real-time transport protocol (SRTP)," RFC 3711 (Proposed Standard), March 2004.

[13] “Doja developer network," http://www.doja-developer.net/

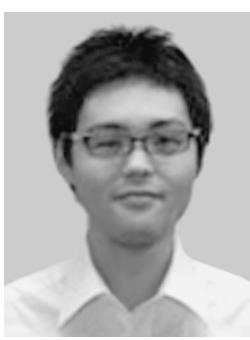

Kenichi Ishibashi received a M.E. degree from the Nara Institute of Science and Technology in 2006 and is currently a Ph.D. candidate at the Nara Institute of Science and Technology. His research interests are mobile networking, transport protocols, and network mobility.

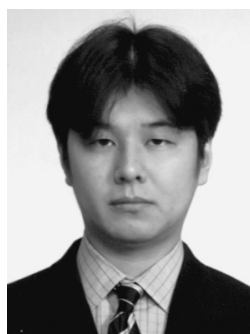

Naoto Morishima received a B.S. degree from Kyoto University in 1996, a M.E. in computer science from Nara Institute of Science and Technology in 1998, and a Ph.D. in computer science from Nara Institute of Science and Technology in 2002. He has been an assistant professor of the Nara Institute of Science and Technology since 2002 .

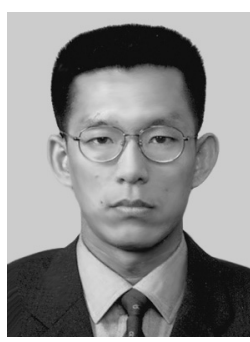

Masayuki Kanbara received his B.E. degree in information technology from Okayama University in 1997 and his Ph.D. degree in information science from the Nara Institute of Science and Technology in 2002. He has been an assistant professor of Nara Institute of Science and Technology since 2002.

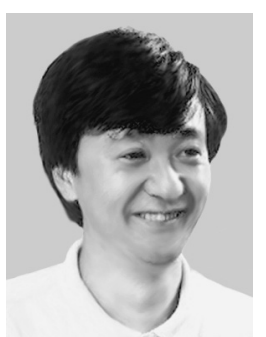

Hideki Sunahara received B.S. and M.S. degrees in electrical engineering from Keio University in 1983 and 1985 and a Ph.D. in computer science from Keio University in 1989 Currently, he is a professor in the Graduate School of Media Design, Keio University. His research focuses on multimedia communication systems, digital libraries, computer architecture, parallel processing, distributed systems, operating systems, and computer networks. $\mathrm{He}$ is a member of ACM, IEEE, Internet Society, JSSST, and IPSJ. He is also a board member of the WIDE project.

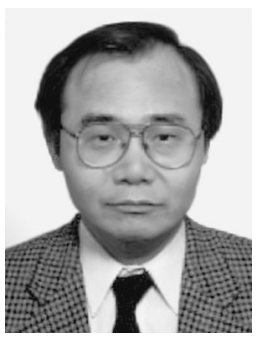

Masami Imanishi received B.M. and M.M. degrees in Neurosurgery from Nara Medical University, in 1979 and 1983, respectively. He received the Ph.D. degree in Radiology from Nara Medical University, in 1985. He was a medical doctor of emergency medical care center, Nara Prefectural Nara Hospital. Currently, he is the assistant director of Nara Prefectural Gojo Hospital. He is also the chief of department of neurosurgery, Nara Prefectural Gojo Hospital and the director of Nara Prefectural Gojo Hospital Nursing School. 